\title{
Communication \\ An Efficient Maritime Target Joint Detection and Imaging Method with Airborne ISAR System
}

\author{
Haodong Li *, Guisheng Liao, Jingwei Xu (D) and Lan Lan \\ National Laboratory of Radar Signal Processing, Xidian University, Xi'an 710071, China; \\ liaogs@xidian.edu.cn (G.L.); jwxu@xidian.edu.cn (J.X.); lanlan@xidian.edu.cn (L.L.) \\ * Correspondence: lihd@stu.xidian.edu.cn; Tel.: +86-155-2963-7271
}

Citation: Li, H.; Liao, G.; Xu, J.; Lan, L. An Efficient Maritime Target Joint Detection and Imaging Method with Airborne ISAR System. Remote Sens. 2022, 14, 193. https://doi.org/ $10.3390 /$ rs14010193

Academic Editors: M. Pilar Jarabo Amores, David de la Mata Moya and Dusan Gleich

Received: 24 November 2021 Accepted: 31 December 2021 Published: 1 January 2022

Publisher's Note: MDPI stays neutral with regard to jurisdictional claims in published maps and institutional affiliations.

Copyright: (C) 2022 by the authors. Licensee MDPI, Basel, Switzerland. This article is an open access article distributed under the terms and conditions of the Creative Commons Attribution (CC BY) license (https:// creativecommons.org/licenses/by/ $4.0 /)$.

\begin{abstract}
In this paper, a joint maritime moving target detection and imaging approach, referred to as the fast inverse synthetic aperture radar (ISAR) imaging approach, based on the multi-resolution space-time adaptive processing (STAP), is proposed to improve the target detection performance and the target imaging efficiency in an airborne radar system. In the target detection stage, the sub-band STAP is introduced to improve the robustness of clutter suppression and to enhance the target output power with the decreased range resolution, by which the coarse estimation of target range-Doppler (R-D) location is obtained as the prior knowledge. In the following target imaging stage, the ISAR imaging is applied in the localized R-D zone surrounding with the target location. However, it is difficult to directly apply ISAR imaging with the conventional R-D algorithm because the slowmoving maritime target cannot be separated from the clutter interference in the Doppler frequency dimension. In this regard, the full-band STAP is applied in the R-D two-dimensional frequency domain for the simultaneous clutter suppression and high-resolution ISAR imaging, in which the envelope alignment and phase compensation are achieved by adaptive match filtering with the target Doppler frequency coarse estimation. Moreover, the reduced-dimension STAP applied in the target-surrounded localized Doppler frequency zone gives facilities for alleviating the computation burden. Simulation results corroborate the effectiveness of the proposed method.
\end{abstract}

Keywords: space-time adaptive processing; maritime moving target detection; inverse synthetic aperture radar; robust clutter suppression; multi-resolution

\section{Introduction}

For airborne battlefield surveillance radars, it is challenging to obtain high-resolution radar images of moving targets in the scenario of strong clutter interference. Inverse synthetic aperture radar (ISAR) imaging is generally applied with a fixed platform by utilizing the relative motion of a non-cooperative target [1-3], which is supposed to be effective in the absence of strong clutter interference. However, in an airborne radar system, the Doppler spread of strong clutter is inevitable due to the geometry-induced spatial-temporal frequency coupling effect. Furthermore, this Doppler spread issue will be aggravated in the airborne ISAR system, because of the large carrier frequency employed for the azimuth Doppler frequency distinction among scatters in the ISAR image. Hence, it is difficult to separate the slow-moving maritime target signal from clutter interference with the conventional pulse-Doppler based radar. Space-time adaptive processing (STAP) algorithms have been widely applied for clutter suppression and low-altitude slow moving target detection in airborne radar systems [4-6], where the weight vector basically consists of a whitening operation followed by a spatial-temporal two-dimensional (2D) match filter. However, it is difficult to apply the STAP directly in the ISAR system. The challenges lie in two aspects: (i) the transmitted bandwidth and the coherent processing interval (CPI) are each increased to improve range-Doppler (R-D) resolutions in the ISAR image, which cause the nonstationary echo signal in regard to the range walking (RW) of target and clutter, 
leading to the unsatisfied requirement of STAP and the corresponding clutter suppression performance degradation [7] and the target coherent accumulation performance loss [8-11], and (ii) the large-scale maritime target becomes an range-extended target in the highresolution ISAR system, leading to target output power loss and to corresponding target detection performance degradation of STAP.

An efficient ISAR imaging method based on the sliding window STAP was proposed in our previous work [12], where the whole CPI is divided into a series of sub-CPIs. Here, the STAP is applied in each sub-CPI to suppress the clutter and to improve the SCNR performance, while the Doppler frequency linear difference among scatters is wellconstrained in the azimuth dimension, contributing to the further ISAR imaging based on the R-D algorithm [13]. However, the decreased DOFs of sub-CPI STAP degrade the output SCNR performance. Hence, under a low signal-to-noise ratio condition, the target detection performance might not satisfy the requirement of the following ISAR imaging. In [14], a combined space-Doppler adaptive ISAR processing (SDAP-ISAR) was proposed to perform adaptive clutter suppression and ISAR imaging. This method performs adaptive match filtering in the Doppler frequency dimension, which achieves a good imaging performance and requires less training samples by introducing the reduceddimension technique within the segmented Doppler frequency zones $[15,16]$. However, the computational complexity is too high to meet the real-time requirement, which is because the calculation of adaptive weight vector and the match filtering need to be implemented for every Doppler frequency pixel sample of every range frequency sample in the ISAR image, and the clutter covariance matrix (CCM) estimation also has to be repeatedly implemented within the whole unambiguity Doppler frequency scope. Furthermore, the SAR imaging stage needs to be involved prior to ISAR imaging. On the basis of the blurred SAR image, further residual motion compensation is required with parametric search in the absence of prior knowledge of the target Doppler frequency, which leads to unaffordable computational complexity.

In this paper, a fast ISAR imaging approach based on the multi-resolution STAP is proposed for airborne ISAR to improve the target detection performance and the imaging efficiency. At the target detection stage, the sub-band STAP is introduced with the subband division technique $[17,18]$, by which the nonstationary echo signal is alleviated in regard to the RW of target and clutter, while the target extending issue is also alleviated. In this regard, the coarse estimation of the target R-D location can be obtained robustly by improving the output SCNR performance of the STAP. However, even with the target R-D location, it is still infeasible to apply ISAR imaging of endo-clutter slow-moving maritime targets with the conventional R-D algorithm. In this regard, the full-band STAP is applied in the R-D 2D frequency domain for clutter suppression and high-resolution ISAR imaging simultaneously, in which envelope alignment and phase compensation are achieved by match filtering with the target Doppler frequency coarse estimation. Moreover, based on the reduced-dimension technique, the adaptive match filtering applied in the localized R-D zone gives facilities for alleviating the computation burden, especially for the computational complexity induced by the redundant CCM estimation within the whole Doppler frequency scope. The novelties and contributions of this paper are summarized as follows.

i It circumvents maritime target detection and clutter suppression performances degradation in the ISAR system, which is achieved by eliminating the RW and target extending issues based on the sub-band STAP;

ii The coarse estimation of target R-D location obtained in the sub-band STAP output is subsequently utilized as a priori knowledge, which gives facilities to the fast full-band STAP applied in the localized R-D zone for the simultaneous clutter suppression and high-resolution ISAR imaging.

The remainder of this paper is organized as follows. Section 2 establishes the signal model. The proposed fast ISAR imaging approach based on the multi-resolution STAP is 
presented in Section 3. Section 4 shows the computer simulations results, and the conclusion is drawn in Section 5.

\section{Signal Model}

Without a loss of generality, Figure 1 shows the geometry of a maritime moving target in an airborne ISAR system. The array radar is employed in side-looking mode, and the velocity of radar is $v_{a}$, along the $X$-axis direction. The linear array is assumed to have $N$ omnidirectional elements with uniform inter-element spacing $d$ along the $X$-axis. $K$ pulses are transmitted during a CPI, the pulse repetitive time (PRT) is defined as $T$, the $O-X Y$ plane is the sea, and the Z-axis is perpendicular to the sea.

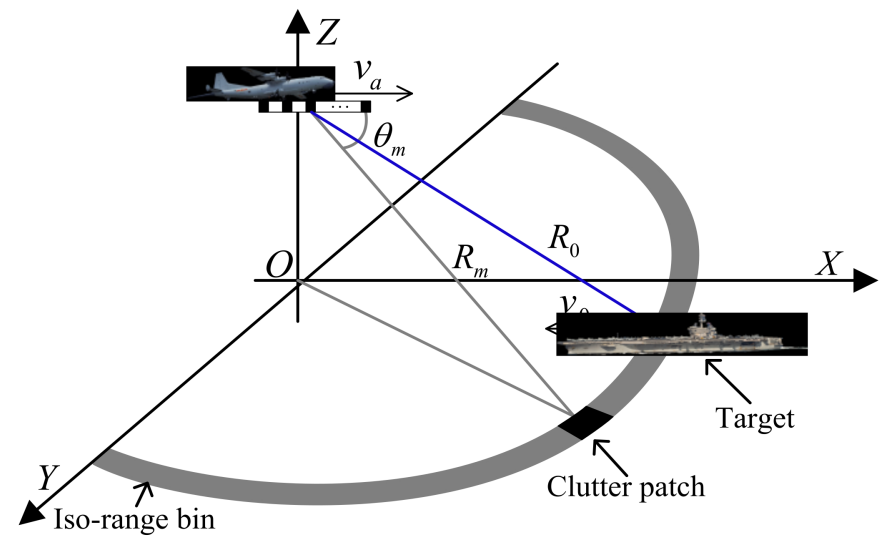

Figure 1. Geometric sketch of airborne ISAR.

The baseband signal of the $k$-th pulse after digital down conversion is written as

$$
x(f, k)=x_{t}(f, k)+x_{c}(f, k)+n(f, k),
$$

where $f$ is the range frequency, $x_{t}(f, k)$ is the range-extended target signal, $x_{c}(f, k)$ is the clutter signal, and $n(f, k)$ is the additive white Gaussian noise (AWGN). Considering that the target consists of $L$ scatter points, the spectrum of the transmitted linear frequency modulation (LFM) signal is $\varphi(f)$, which is distributed within the whole transmitted bandwidth. The $x_{t}(f, k)$ can be expressed based on the turntable model, that is,

$$
\begin{gathered}
x_{t}(f, k)=\sum_{l=1}^{L} \xi_{l} \varphi(f) e^{-j \frac{4 \pi\left(f+f_{c}\right)}{c} R_{l, k},} \\
R_{l, k}=R_{0, k}+y_{2, l}+\Omega_{e f f, t} y_{1, l} T(k-1),
\end{gathered}
$$

where $\xi_{l}$ denotes the coefficient of scatter point; $R_{0, k}$ denotes the slant range of the reference scatter point in the $k$-th pulse; $y_{1, l}$ and $y_{2, l}$ denote the azimuth and range coordinates of the $l$-th scatter point on the imaging project plane, respectively; and $\Omega_{e f f, t}$ denotes the effective rotation vector projected on a plane orthogonal to the radar line-of-sight due to the relative motion of the target.

Considering that the clutter signal can be regarded as the summation of the echo reflected from many clutter patches in the scene, and the clutter scattering characteristic is assumed to be stationary within the whole CPI for the sake of simplicity. $x_{c}(f, k)$ can be expressed as

$$
x_{c}(f, k)=\sum_{m=1}^{M} \xi_{m} \varphi(f) e^{-j \frac{4 \pi\left(f+f_{c}\right)}{c} R_{m, k}},
$$

where $R_{m, k}$ is the slant range of the $m$-th clutter patch corresponding to the $k$-th pulse, and $\xi_{m}$ is the stationary scattering coefficient of clutter patch. Note that $\xi_{m}$ will be dynamic and stochastic under the nonstationary electromagnetic scattering condition, leading to the 
nonhomogeneous training sample issue. Moreover, (2) and (4) are the received echo signal of a single channel in the joint slow-time and range frequency domain, respectively, and the multi-channels echo signal is obtained with the phase modulation of the inter-channel wave path-difference, which is expressed as

$$
\begin{gathered}
\mathbf{x}(f, k)=\mathbf{x}_{\mathbf{t}}(f, k)+\mathbf{x}_{\mathbf{c}}(f, k)+\mathbf{n}(f, k) \\
=x_{t}(f, k) \mathbf{s}_{\mathbf{s}}\left(f_{s, 0, k}\right)+\sum_{m=1}^{M} \xi_{m} \varphi(f) e^{-j \frac{4 \pi\left(f+f_{c}\right)}{c} R_{m, k}} \mathbf{s}_{\mathbf{s}}\left(f_{s, m, k}\right)+\mathbf{n}(f, k) \\
\mathbf{s}_{s}\left(f_{s}\right)=\left[1, e^{j 2 \pi f_{s}}, \ldots, e^{j 2 \pi f_{s}(N-1)}\right]^{T},
\end{gathered}
$$

where $\mathbf{s}_{s}\left(f_{s}\right)$ denotes the spatial steering vector, and $f_{s, 0, k}=\frac{f_{c} d \cos \left(\theta_{0, k}\right)}{c}$ and $f_{s, m, k}=\frac{f_{c} d \cos \left(\theta_{m, k}\right)}{c}$ denote the corresponding normalized spatial frequencies in the $k$-th pulse for target and clutter, respectively. After match filtering, it yields,

$$
\mathbf{x}(f, k, B)=\operatorname{rect}\left(\frac{f}{B}\right)\left(\sum_{l=1}^{L} \xi_{l} e^{-j \frac{4 \pi\left(f+f_{c}\right)}{c} R_{l, k}} \mathbf{s}_{\mathbf{s}}\left(f_{s, 0, k}\right)+\sum_{m=1}^{M} \xi_{m} e^{-j \frac{4 \pi\left(f+f_{c}\right)}{c} R_{m, k}} \mathbf{s}_{\mathbf{s}}\left(f_{s, m, k}\right)\right)+\mathbf{n}(f, k),
$$

where $B$ is the full-band width. By utilizing the sub-band division technique [18], it can be truncated into the sub-band signal $\mathbf{x}\left(f, k, B_{s u b}\right)$, corresponding to the sub-band width $B_{s u b}$. By stacking echoes corresponding to all $K$ pulses into a vector in the joint range-frequency and slow-time domain, it yields

$$
\mathbf{x}\left(f, B_{\text {sub }}\right)=\left[\mathbf{x}^{T}\left(f, 1, B_{\text {sub }}\right), \mathbf{x}^{T}\left(f, 2, B_{\text {sub }}\right), \ldots, \mathbf{x}^{T}\left(f, K, B_{\text {sub }}\right)\right]^{T} .
$$

Implementing inverse fast Fourier transform (IFFT) with respect to range frequency, it yields

$$
\begin{aligned}
\mathbf{x}\left(\tau, k, B_{\text {sub }}\right) & =\sum_{l=1}^{L} \xi_{l} s_{t}\left(R_{l . k}, \tau\right) \mathbf{s}_{\mathbf{s}}\left(f_{s, 0, k}\right)+\sum_{m=1}^{M} \xi_{m} s_{t}\left(R_{m, k}, \tau\right) \mathbf{s}_{\mathbf{s}}\left(f_{s, m, k}\right) \\
& =\sum_{l=1}^{L} \xi_{l} G_{P} \sin \mathrm{c}\left[B_{s u b}\left(\tau-2 R_{l, k} / c\right)\right] e^{-j \frac{4 \pi f_{c} R_{l, k}}{c}} \mathbf{s}_{\mathbf{s}}\left(f_{s, 0, k}\right) \\
& +\sum_{m=1}^{M} \xi_{m} G_{P} \operatorname{sinc}\left[B_{s u b}\left(\tau-2 R_{m, k} / c\right)\right] e^{-j \frac{4 \pi f_{c} R_{m, k}}{c}} \mathbf{s}_{\mathbf{s}}\left(f_{s, m, k}\right)+\mathbf{n}(\tau, k)
\end{aligned}
$$

where $G_{P}$ denotes the range compression gain. It is observed from (9) that the range resolution of the envelope decreases with $B_{s u b}$. It should be noted that the multichannel echo signal $\mathbf{x}\left(\tau, k, B_{\text {sub }}\right) \in \mathbb{C}^{N \times 1}$ is a sample of channels for a given fast-time and slow-time instance. By stacking echoes corresponding to all $K$ pulses into a vector, it yields

$$
\mathbf{x}\left(\tau, B_{\text {sub }}\right)=\left[\mathbf{x}^{T}\left(\tau, 1, B_{\text {sub }}\right), \mathbf{x}^{T}\left(\tau, 2, B_{\text {sub }}\right), \ldots, \mathbf{x}^{T}\left(\tau, K, B_{\text {sub }}\right)\right]^{T},
$$

where $\mathbf{x}\left(\tau, B_{\text {sub }}\right) \in \mathbb{C}^{N K \times 1}$ denotes the spatial-temporal 2D data corresponding to a particular time instance, which includes the possible target, clutter, and AWGN.

\section{ISAR Imaging Based on the Multi-Resolution STAP}

In this section, the proposed fast ISAR imaging approach based on the multi-resolution STAP is introduced specifically, which can be categorized into two stages in sequence. In the first stage, the sub-band STAP is applied for robust clutter suppression and range-extended target detection, through which the coarse estimation of the target R-D location is derived with the SCNR-enhanced STAP output. However, it is still far from enough to apply ISAR imaging with the conventional R-D algorithm, because the slow-moving maritime target is infeasible to be separated from the Doppler-spread clutter in the airborne radar system. In the second stage, by utilizing the target Doppler frequency coarse estimation and the 
dimension-reduced STAP technique, the full-band adaptive match filter is designed for the simultaneous clutter suppression and motion compensation in the target-surrounded localized Doppler frequency zone, which gives facilities for the following fast ISAR imaging of the endo-clutter maritime target in the localized R-D zone. The flowchart of the proposed method is shown in Figure 2.

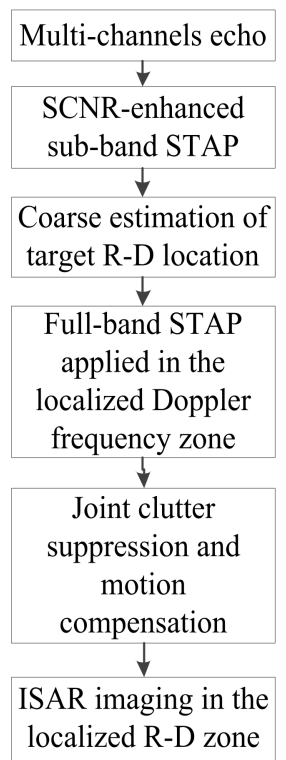

Figure 2. Flowchart of the proposed method.

\subsection{Robust Clutter Suppression with Sub-Band STAP}

In the airborne ISAR system, nonhomogeneous clutter is inevitable under the condition of high range resolution and long CPI, which causes inaccurate statistical methodologiesbased CCM estimation because of the RW and nonstationary scattering issues of clutter [7], that is,

$$
\hat{\mathbf{R}}_{C N, B}=\frac{1}{\|\mathbf{\Omega}\|_{0}} \sum_{l \in \mathbf{\Omega}} \mathbf{x}(l, B) \mathbf{x}(l, B)^{\mathrm{H}},
$$

where $\Omega$ denotes the range of secondary data surrounding the CUT, and the superscript $(\cdot)^{\mathrm{H}}$ denotes the conjugate transpose operator. According to the well-known Reed-MallettBrennan rule for statistical-methodology-based STAP, the output SCNR loss is less than $3 \mathrm{~dB}$, where the number of independent and identically distributed (I.I.D) training samples is more than twice of the system's degrees-of-freedom (DOFs) [19]. To tackle the clutter suppression performance degradation induced by the clutter RW, it is feasible to eliminate the clutter RW by decreasing the range resolution. However, as seen from (9), the dependence of the adjacent training samples increases dramatically with the decreased range resolution, which corrupts the I.I.D characteristic of the training samples and causes CCM estimation errors due to insufficient training samples. Hence, we carried out the direct data domain approach [20-22] to address this problem of insufficient training samples, and the obtained advantages lie in two aspects: (i) the nonstationary scattering issue of the clutter will be mitigated within the shorter CPI, and (ii) the insufficient training samples induced by the decreased range resolution is addressed, and the I.I.D training samples are supplemented by taking advantage of the long CPI in the ISAR system.

We further derive the weight vector of the sub-band STAP as

$$
\mathbf{w}_{m_{2}, m_{1}}=\mu \hat{\mathbf{R}}_{C N, B_{s u b}}^{-1} \mathbf{s}_{s, t}^{H}\left(f_{d, m_{2}}, f_{s, m_{1}}\right), \quad m_{2}=0,1, \ldots, M_{2}-1, \quad m_{1}=0,1, \ldots, M_{1}-1,
$$




$$
\begin{gathered}
\mu=\frac{1}{\mathbf{s}_{s, t}^{H}\left(f_{d, m_{2}}, f_{s, m_{1}}\right) \hat{\mathbf{R}}_{C N, B_{s u b}}^{-1} \mathbf{s}_{s, t}\left(f_{d, m_{2}}, f_{s, m_{1}}\right)}, \\
\mathbf{s}_{s, t}\left(f_{d, m_{2}}, f_{s, m_{1}}\right)=\mathbf{s}_{t}\left(f_{d, m_{2}}\right) \otimes \mathbf{s}_{s}\left(f_{s, m_{1}}\right),
\end{gathered}
$$

where $\mathbf{w}_{m_{2}, m_{1}} \in \mathbb{C}^{N K \times 1}$ is the STAP weight vector corresponding to the normalized sampling frequency pair $\left(f_{d, m_{2}}, f_{s, m_{1}}\right) ; M_{2}$ and $M_{1}$ are the uniform sampling frequency grid point numbers of temporal and spatial dimensions, respectively; $\mathbf{s}_{s, t}\left(f_{d, m_{2}}, f_{s, m_{1}}\right) \in \mathbb{C}^{N K \times 1}$ denotes the corresponding spatial-temporal 2D steering vector; and $\mu$ is the normalized factor. $\mathbf{s}_{t}\left(f_{d, m_{2}}\right)$ is the temporal steering vector corresponding to the normalized Doppler sampling frequency $f_{d, m_{2}}$, that is,

$$
\mathbf{s}_{t}\left(f_{d, m_{2}}\right)=\left[1, e^{j 2 \pi f_{d, m_{2}}}, \ldots, e^{j 2 \pi f_{d, m_{2}}(K-1)}\right]^{\mathrm{T}} .
$$

In addition to the robust clutter suppression performance, by alleviating the target extending and RW issues of the maritime target with the decreased range resolution, the sub-band STAP can also improve the range-extended target detection performance with the enhanced SCNR performance. Under the condition of in-phase magnitude-phase superposition of range-compressed complex envelopes among adjacent scattering points, the range-extended target detection performance loss is alleviated by contributing the output powers of the scattering points to the corresponding synthesized output power. However, this mechanism is dependent on the dynamic and stochastic scattering coefficients in practice, which is discussed specifically in 18. As for the target output power loss induced by the RW in the match filtering, the decreased range resolution can also alleviate the target RW issue without any preprocessing. Hence, it is feasible to obtain the coarse estimation of the target R-D location with the target detection technique [23] based on the SCNR-enhanced STAP output, which gives facilities for further ISAR imaging in the localized R-D zone.

\subsection{Efficient ISAR Imaging with the Full-Band STAP}

Based on the coarse estimation of the target R-D location obtained from the SCNRenhanced sub-band STAP output, the efficient ISAR imaging can be applied subsequently in the localized R-D zone, which gives facilities to computational complexity alleviation. However, only the target R-D location is far from enough for the efficient ISAR imaging of maritime targets with the conventional R-D algorithm, because the endo-clutter slowmoving maritime target is infeasible to be extracted out in the Doppler frequency dimension. Hence, we applied the full-band STAP in the R-D 2D frequency domain for simultaneous clutter suppression and high-resolution ISAR imaging, in which the target Doppler frequency coarse estimation was utilized for the envelope alignment and phase compensation by match filtering in the target-surrounded localized Doppler frequency zone.

The coarse estimation of the normalized Doppler frequency and normalized spatial frequency are obtained from the target detection output in the R-D domain, which are assumed to be $f_{d, 0}$ and $f_{s, 0}$, respectively. Considering that the target Doppler ambiguity factor is small enough under the condition of a slow-moving maritime target, the unambiguous Doppler frequency can be derived with less searching operations, that is,

$$
f_{d, t}=f_{d, 0}+\eta_{a m b}
$$

where $\eta_{a m b}$ denotes the target Doppler ambiguity factor estimation. Combined with digital beam forming (DBF), range alignment and phase compensation can be achieved simultaneously with the match filtering in the joint range frequency and slow-time domain, that is,

$$
\mathbf{x}_{f}=\sum_{n=1}^{N} \mathbf{F}\left[\mathbf{x}_{n}(f, B) \odot \mathbf{s}_{f, k}^{*}\left(f_{d, t}\right)\right] \times e^{-j 2 \pi f_{s, 0}(n-1)},
$$




$$
\mathbf{s}_{f, k}\left(f_{d, t}\right)=\left[1, e^{j 2 \pi f_{d, t} \frac{f+f_{c}}{f_{c}}}, \ldots, e^{j 2 \pi f_{d, t} \frac{f+f_{c}}{f_{c}}(K-1)}\right]^{\mathrm{T}},
$$

where $\mathbf{x}_{n}(f, B) \in \mathbb{C}^{K \times 1}$ denotes the echo signal extracted from the corresponding rows of $\mathbf{x}(f, B)$, representing the $n$-th single channel echo signal; $\odot$ denotes the Hadamard product operator; $(\cdot)^{*}$ denotes the conjugate operator; $\mathbf{x}_{f} \in \mathbb{C}^{K \times 1}$ denotes the Doppler frequency samples vector corresponding to the range frequency sample $f$; and $\mathbf{F}$ denotes the discrete Fourier transform matrix. Stack $\mathbf{x}_{f}$ as columns into the matrix $\mathbf{X}_{f}$, where $f$ spans the whole range frequency scope $\left[-\frac{f_{s}}{2}, \frac{f_{s}}{2}\right)$. On this basis, by implementing inverse fast Fourier transform with respect to the range dimension, the ISAR image can be further obtained in the zero-Doppler frequency zone, that is,

$$
\mathbf{X}_{\text {imag }}=\mathcal{F}_{f}^{-1}\left[\mathbf{X}_{f}\right]
$$

where $\mathcal{F}_{f}^{-1}[\cdot]$ denotes the inverse Fourier transform with respect to the range frequency dimension, and it is referred to as conventional R-D algorithm-based ISAR imaging. However, it is infeasible to directly apply ISAR imaging for the endo-clutter slow-moving maritime target, which calls for the joint adaptive clutter suppression and ISAR imaging approach, accordingly. Inspired by the combination of STAP and R-D algorithm-based ISAR imaging [14], we propose a more efficient maritime ISAR imaging algorithm based on the SCNR-enhanced sub-band STAP output as follows.

According to the convolution theorem, the (17) can be expressed in the Doppler frequency domain as

$$
\mathbf{x}_{f}=\sum_{n=1}^{N} \mathbf{F}\left[\mathbf{x}_{n}(f, B)\right] * \mathbf{F}\left[\mathbf{s}_{f, k}^{*}\left(f_{d, t}\right)\right] \times e^{-j 2 \pi f_{s, 0}(n-1)},
$$

where $*$ denotes the convolution operator. The $k$-th element of $\mathbf{x}_{f} \in \mathbb{C}^{K \times 1}$ can be further derived as

$$
\begin{gathered}
\mathbf{x}_{f}(k)=\sum_{n=1}^{N} \mathbf{g}_{f, f_{d}}^{H}\left(f_{d, k}\right) \mathbf{x}_{n, f_{d}}(f) e^{-j 2 \pi f_{s, 0}(n-1)}, \\
\mathbf{x}_{n, f_{d}}(f)=\mathbf{F}\left[\mathbf{x}_{n}(f, B)\right], \\
\mathbf{g}_{f, f_{d}}\left(f_{d, k}\right)=\left[\mathbf{s}_{f, f_{d}}(-k), \mathbf{s}_{f, f_{d}}(1-k), \ldots, \mathbf{s}_{f, f_{d}}(K-1-k)\right]^{T}, \\
\mathbf{s}_{f, f_{d}}=\mathbf{F}\left[\mathbf{s}_{f, k}\left(f_{d, t}\right)\right] .
\end{gathered}
$$

where $f_{d, k}$ denotes the $k$-th Doppler frequency pixel sample in the ISAR image. Equation (21) can be further simplified into the spatial-temporal 2D match filtering, that is,

$$
\begin{gathered}
\mathbf{x}_{f}(k)=\mathbf{g}_{f_{s}, f_{d}}^{H}\left(f_{d, k}, f_{s, 0}\right) \mathbf{x}_{f_{d}}(f), \\
\mathbf{g}_{f_{s}, f_{d}}\left(f_{d, k}, f_{s, 0}\right)=\mathbf{g}_{f, f_{d}}\left(f_{d, k}\right) \otimes \mathbf{s}_{s}\left(f_{s, 0}\right),
\end{gathered}
$$

where the multi-channel echo signal $\mathbf{x}_{f_{d}}(f) \in \mathbb{C}^{N K \times 1}$ is stacked by $\left\{\mathbf{x}_{n, f_{d}}(f) \mid n=1,2, \cdots, N,\right\}$, referred to as the Doppler frequency domain representation of $\mathbf{x}(f, B)$. On this basis, the adaptive clutter suppression is further combined with the match filtering in (25), which utilizes the STAP technique specifically, that is,

$$
\begin{gathered}
\mathbf{x}_{f}(k)=\mathbf{w}_{f, f_{d}}^{H}\left(f_{d, k}\right) \mathbf{x}_{f_{d}}(f), \\
\mathbf{w}_{f, f_{d}}\left(f_{d, k}\right)=\hat{\mathbf{R}}_{C N, f_{d}}^{-1} \mathbf{g}_{f_{s}, f_{d}}\left(f_{d, k}, f_{s, 0}\right),
\end{gathered}
$$




$$
\hat{\mathbf{R}}_{C N, f_{d}}=\frac{1}{\|\boldsymbol{\Omega}\|_{0}} \sum_{l \in \boldsymbol{\Omega}} \mathbf{x}_{f_{d}}(l, B) \mathbf{x}_{f_{d}}(l, B)^{\mathrm{H}},
$$

where $\mathbf{x}_{f_{d}}(l, B)$ is the Doppler frequency domain representation of $\mathbf{x}(\tau, B)$, and the $\Omega$ can be directly obtained without parametric search according to the target range location coarse estimation in the SCNR-enhanced sub-band STAP output. It should be noted that the adaptive clutter suppression is available to $\mathbf{x}_{f_{d}}(f)$ with different $f$ in (27). This is because, as shown from the clutter signal in (7), the $f$-dependent phase modulation term $e^{-j \frac{4 \pi f}{c} R_{m, k}}$ is negligible compared with $e^{-j \frac{4 \pi f_{c} R_{m, k}}{c}}$ for a sample of pulses at a specific range frequency sample $f$, where the $f$ is smaller than $f_{c}$. Hence, by following the concept of the negligible phase term involved in the frequency diversity-based approaches [24-26], we can consider that the Doppler frequency of the clutter signal is not coupled with the range frequency, which gives facilities for the consistent CCM estimation with $\mathbf{x}_{f_{d}}(l, B)$ for clutter suppression in $\mathbf{x}_{f_{d}}(f)$.

Taking the coarse estimation of the target R-D location as the prior knowledge, the computational complexity of the aforementioned adaptive match filtering can be further dramatically decreased. Specifically, the target range location can be utilized to reduce the match filtering scope in the range dimension, which represents the lower range frequency samples of $\mathbf{x}_{f_{d}}(f)$ to be filtered in (27). As for the target Doppler frequency location, it can be utilized to reduce the number of Doppler frequency pixel samples in the ISAR image, through which the $f_{d, k}$ in (27) can be selected from the zero-Doppler-frequency localized zone instead of the whole Doppler frequency dimension. Furthermore, with the reduced-dimension technique of STAP, both $\mathbf{w}_{f, f_{d}}\left(f_{d, k}\right)$ and $\mathbf{x}_{f_{d}}(f)$ can be segmented into the target-surrounded localized Doppler frequency zone, referred to as $\mathbf{w}_{f, f_{d, t}}\left(f_{d, k}\right)$ and $\mathbf{x}_{f_{d, t}}(f)$, respectively, i.e.,

$$
\begin{gathered}
\mathbf{w}_{f, f_{d, t}}\left(f_{d, k}\right)=\hat{\mathbf{R}}_{C N, f_{d, t}}^{-1} \mathbf{g}_{f_{s}, f_{d, t}}\left(f_{d, k}, f_{s, 0}\right), \\
\mathbf{g}_{f_{s}, f_{d, t}}\left(f_{d, k}, f_{s, 0}\right)=\mathbf{g}_{f, f_{d, t}}\left(f_{d, k}\right) \otimes \mathbf{s}_{s}\left(f_{s, 0}\right), \\
\mathbf{g}_{f, f_{d, t}}\left(f_{d, k}\right)=\left[\mathbf{s}_{f, f_{d}}\left(\mathbf{f}_{d, t, i n d}(1)-k\right), \mathbf{s}_{f, f_{d}}\left(\mathbf{f}_{d, t, i n d}(2)-k\right), \ldots, \mathbf{s}_{f, f_{d}}\left(\mathbf{f}_{d, t, i n d}\left(K_{s u b}\right)-k\right)\right]^{T},
\end{gathered}
$$

where $\mathbf{f}_{d, t, \text { ind }} \in \mathbb{C}^{K_{\text {sub }} \times 1}$ denotes the Doppler frequency sample orders within the targetsurrounded zone, and $\mathbf{x}_{f_{d, t}}(f)$ denotes the echo signal in the same target-surrounded localized Doppler frequency zone. Compared with the existing joint clutter suppression and ISAR imaging method proposed in [14], the adaptive match filtering voids being applied in the whole unambiguity Doppler scope, which reduces the computational complexity burden dramatically.

\section{Simulation Results}

In this section, numerical examples are provided to assess the performance of the proposed fast ISAR imaging approach based on the multi-resolution STAP, where strong clutter interference and AWGN are in the side-looking airborne radar system. The simulation parameters are listed in Table 1. It is obviously that the values of carrier frequency, transmitted bandwidth and CPI length are all consistent with the general requirements of ISAR system, which satisfy the R-D 2D high resolution performance demand in the ISAR imaging. Moreover, the target is an aircraft carrier located with the DOA angle of 30 degree with respect to the platform velocity. The ship's own rotation driven by waves can also be contributed to the ISAR imaging based on Doppler-estimation based methods [27], in which the rotation vector projected on $\Omega_{e f f, t}$ is synthesized by the geometry configuration variation and the target's own rotation, simultaneously. 


\subsection{SCNR-Enhanced Sub-Band STAP}

In this part, the simulation results of range-extended maritime target detection are carried out to validate the effectiveness in the sub-band STAP stage of the proposed method. To this end, the CPI length is truncated to 512 PRT to avoid the nonstationary scattering issue of the clutter training sample, and the sub-band width is decreased to $10 \mathrm{MHz}$ for alleviating the RW and target extending issues, at a reasonable cost of range compression gain loss. Figure 3a shows the echo spectrum distributed in the R-D domain. It is observed that the endo-clutter slow-moving maritime target cannot be recognized without clutter suppression, which validates that the slow-moving maritime target is infeasible to be separated from the clutter interference with the conventional R-D algorithm. Figure $3 b$ shows the conventional full-band STAP output in the R-D domain. It is observed that the clutter suppression performance suffers from the clutter RW issue in the high-resolution ISAR system, which leads to target detection performance loss, correspondingly. Figure $3 \mathrm{c}$ shows the sub-band STAP output in the target detection stage of the proposed method. It is observed that the clutter suppression performance is improved significantly, which gives facilities to robust target detection and the corresponding R-D location estimation. In order to numerically evaluate the target detection performance advantage of the sub-band STAP, the SCNR performance evaluation was applied to 200 Monte-Carlo experiments, as listed in the Table 2.

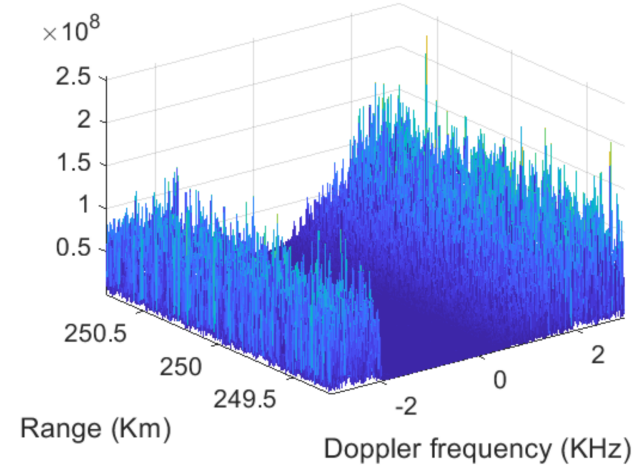

(a)

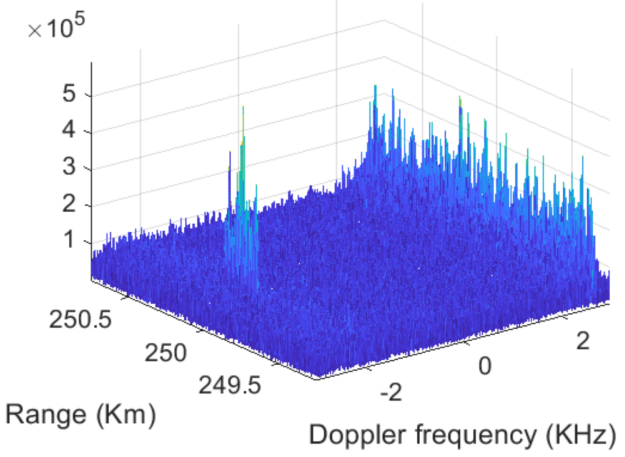

(b)

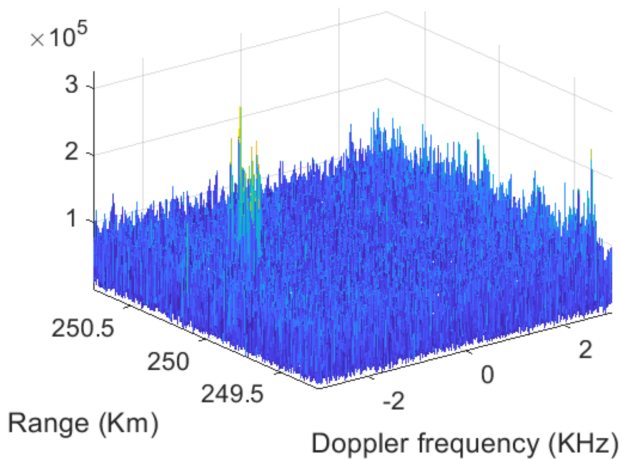

(c)

Figure 3. Simulation results of multi-resolution STAP. (a) Echo spectrum without clutter suppression in the R-D domain. (b) Full-band STAP output in the R-D domain. (c) Sub-band STAP output in the R-D domain.

\subsection{Efficient ISAR Imaging with Full-Band STAP}

In this part, the R-D coarse estimation derived from the SCNR-enhanced sub-band STAP output is utilized as the prior knowledge for motion compensation, through which the range alignment and phase compensation are achieved for the further R-D algorithmbased ISAR imaging. To this end, the full-band and full-CPI are utilized in the ISAR system 
to improve the R-D 2D resolutions of the ISAR image. Figure 4a shows the sliding window STAP-based ISAR imaging result, in which the sub-CPI length is 24 PRT and the sliding window interval is 1 PRT. It is observed that the ISAR image of the maritime target can be formed under the strong clutter interference in general. However, there is still residual clutter interference in the ISAR image, leading to ISAR imaging performance corruption. This is because the DOFs of the sliding window STAP are decreased within the shorter CPI, inevitably degrading the clutter suppression the performance. Figure $4 \mathrm{~b}$ shows the ISAR imaging result of the proposed method based on the multi-resolution STAP. It is observed that the ISAR image of the maritime target is well-focused with the adaptive match filtering in the localized R-D zone, which achieves the clutter suppression and motion compensation simultaneously. It should also be noted that the aforementioned SDAP-ISAR approach can also obtain the same maritime target ISAR image with the proposed method. However, it is achieved at the cost of unaffordable computational complexity, because of the adaptive match filtering applied in the whole Doppler frequency dimension and the parametric search applied for the residual motion compensation after blurred SAR imaging. As for the SDAP-ISAR approach, as the computational complexity induced by the parametric search and the Doppler frequency pixel samples in the ISAR image are non-deterministic, the specific computational complexity analysis and computational consuming time comparison are not evaluated numerically.

Table 1. Simulation Parameters.

\begin{tabular}{cccc}
\hline Parameter & Value & Parameter & Value \\
\hline Carrier frequency & $10 \mathrm{GHz}$ & Platform height & $10 \mathrm{~km}$ \\
Full bandwidth & $50 \mathrm{MHz}$ & Platform velocity & $150 \mathrm{~m} / \mathrm{s}$ \\
CPI & $0.7 \mathrm{~s}$ & Array number & 8 \\
PRF & $6 \mathrm{kHz}$ & Pulse width & $10 \mu \mathrm{s}$ \\
Slant range of target & $250 \mathrm{~km}$ & clutter-to-noise ratio & $40 \mathrm{~dB}$ \\
SNR & $-10 \mathrm{~dB}$ & Target velocity & $19.5 \mathrm{~m} / \mathrm{s}$ \\
Sub-band width & $10 \mathrm{MHz}$ & Target rotation vector & $0.0175 \mathrm{rad} / \mathrm{s}$ \\
\hline
\end{tabular}

Table 2. Target detection performances.

\begin{tabular}{cc}
\hline Methods & Output SCNR \\
\hline Conventional full-band STAP & $1.42 \mathrm{~dB}$ \\
Sub-band STAP & $9.63 \mathrm{~dB}$ \\
\hline
\end{tabular}

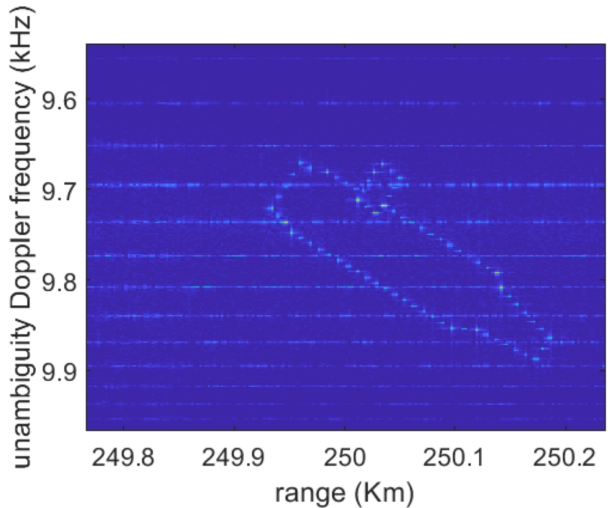

(a)

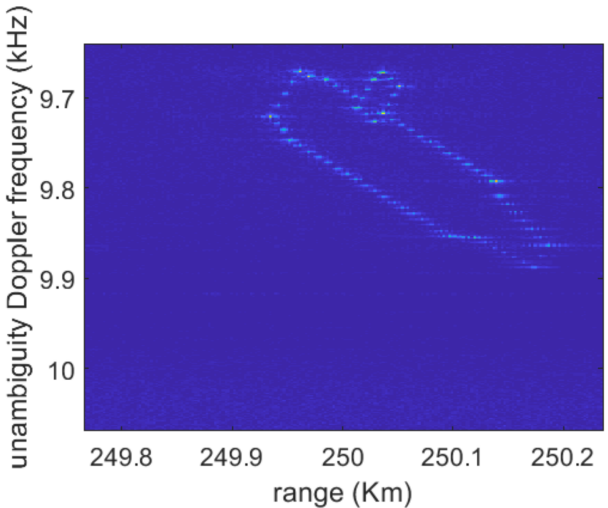

(b)

Figure 4. Simulation results of ISAR imaging. (a) Sliding window STAP-based ISAR imaging result. (b) ISAR imaging result of the proposed method. 


\section{Conclusions}

The maritime target imaging performance degrades dramatically in a strong clutter environment, which is an inevitable problem for the airborne ISAR system. To tackle this issue, a joint maritime moving target detection and imaging approach, referred to as the fast ISAR imaging approach based on the multi-resolution STAP, has been proposed for the airborne radar. By applying the sub-band STAP in the target detection stage, the clutter suppression performance degradation and the range-extended target detection performance loss are circumvented in the high-resolution ISAR system, which gives facilities to target detection performance improvement and robust target R-D location coarse estimation. At the target imaging stage, on the basis of the detected target location, the adaptive match filter is designed for simultaneous clutter suppression and motion compensation, followed by the R-D algorithm-based ISAR imaging. In this stage, by applying reduced-dimension full-band STAP in the target-surrounded localized Doppler frequency zone, the ISAR image is fast derived in the target-surrounded R-D zone without any parametric search. The computer simulation results have demonstrated the effectiveness of the proposed method in maritime target ISAR imaging under the presence of clutter interference.

Possible future studies should focus on the experimental measured data-based validation of the proposed method. Specifically, the robustness of the proposed method should also be assessed under the dynamic and stochastic sea conditions in practice. Furthermore, the forward-looking airborne ISAR imaging might also be taken into consideration.

Author Contributions: Conceptualization, H.L. and J.X.; methodology, H.L.; software, H.L.; validation, H.L., J.X. and L.L.; formal analysis, H.L.; investigation, H.L.; resources, G.L.; data curation, J.X.; writing - original draft preparation, H.L.; writing-review and editing, J.X.; visualization, J.X.; supervision, L.L.; project administration, G.L.; funding acquisition, G.L. All authors have read and agreed to the published version of the manuscript.

Funding: This work was supported in part by the National Nature Science Foundation of China (NSFC) under grants 61931016, 62071344, and 62101402, and the China Postdoctoral Science Foundation under grants 2021TQ0261, 2021M702547.

Conflicts of Interest: The authors declare no conflict of interest.

\section{References}

1. Lv, X.; Xing, M.; Wan, C.; Zhang, S. ISAR imaging of maneuvering targets based on the range centroid Doppler technique. IEEE Trans. Image Process. 2010, 19, 141-153. [PubMed]

2. Huang, P.; Liao, G.; Xia, X.-G.; Yang, Z.; Ma, J. A novel algorithm for ISAR imaging based on parameter estimation of cubic phase signal. In Proceedings of the 2016 CIE International Conference on Radar, Guangzhou, China, 10-13 October 2016; pp. 1220-1224.

3. Chen, V.; Martorella, M. Inverse Synthetic Aperture Radar Imaging: Principles, Algorithms and Applications; Institution of Engineering and Technology: Stevenage, UK, 2014.

4. Melvin, W.L. A stap overview. IEEE Aerosp. Electron. Syst. Mag. 2004, 19, 19-35. [CrossRef]

5. Guerci, J.R. Space-Time Adaptive Processing for Radar; Artech House: Norwood, MA, USA, 2003.

6. Ward, J. Space-Time Adaptive Processing for Airborne Radar; Lincoln Lab, MIT: Lexington, MA, USA, 1994.

7. Hu, J.; Tung, W.-W.; Gao, J. A New Way to Model Nonstationary Sea Clutter. IEEE Signal Process. Lett. 2009, 16, 129-132. [CrossRef]

8. Chen, X.; Huang, Y.; Liu, N.; Guan, J.; He, Y. Radon-fractional ambiguity function-based detection method of low-observable maneuvering target. IEEE Trans. Aerosp. Electron. Syst. 2015, 51, 815-833. [CrossRef]

9. Wu, W.; Wang, G.H.; Sun, J.P. Polynomial Radon-Polynomial Fourier transform for near space hypersonic maneuvering target detection. IEEE Trans. Aerosp. Electron. Syst. 2018, 54, 1306-1322. [CrossRef]

10. Xu, J.; Yu, J.; Peng, Y.; Xia, X.-G. Radon-Fourier transform for radar target detection, (I): Generalized Doppler filter bank. IEEE Trans. Aerosp. Electron. Syst. 2011, 47, 1186-1202. [CrossRef]

11. Xu, J.; Zhou, X.; Qian, L.; Xia, X.; Long, T. Hybrid integration for highly maneuvering radar target detection based on generalized radon-fourier transform. IEEE Trans. Aerosp. Electron. Syst. 2016, 52, 2554-2561. [CrossRef]

12. Li, H.; Liao, G.; Xu, J. An efficient ISAR imaging method based on sliding window STAP. In Proceedings of the IEEE 11th Sensor Array and Multichannel Signal Processing Workshop, Hangzhou, China, 8-11 June 2020.

13. Berizzi, F.; Dalle Mese, E.; Diani, M.; Martorella, M. High-Resolution ISAR Imaging of Maneuvering Targets by Means of the Range Instantaneous Doppler Technique: Modeling and Performance Analysis. IEEE Trans. Image Process. 2001, 10, 1880-1890. [CrossRef] [PubMed] 
14. Alessio, B.; Macro, M.; Douglas, A.G.; Fabrizio, B. Space-Doppler adaptive processing for radar imaging of moving targets masked by ground clutter. IET Radar Sonar Navig. 2014, 9, 712-726.

15. Wang, H.; Cai, L. On adaptive spatial-temporal processing for airborne surveillance radar systems. IEEE Trans. Aerosp. Electron. Syst. 1994, 30, 660-670. [CrossRef]

16. Dipietro, R.C. Extended factored space-time processing for airborne radar systems. In Proceedings of the 26th Asilomar Conference on Signals, Systems and Computing, Pacific Grove, CA, USA, 26-28 October 1992; pp. 425-430.

17. Delphine, C.-M.; Jens, K.; Andreas, R.B. Airborne SAR moving target signatures and imagery based on LVD. IEEE Trans. Geosci. Remote Sens. 2008, 46, 3019-3030.

18. Li, H.; Liao, G.; Xu, J.; Zeng, C. Multi-Resolution STAP for Enhanced Ultra-Low-Altitude Target Detection. Remote Sens. 2021 13, 4212. [CrossRef]

19. Reed, I.S.; Mallett, J.D.; Brennan, L.E. Rapid convergence rate in adaptive arrays. IEEE Trans. Aerosp. Electron. Syst. 1974, 10, 853-863. [CrossRef]

20. Sarkar, T.K. A deterministic least-squares approach to space-time adaptive processing (STAP). IEEE Trans. Antennas Propag. 2001, 49, 91-103. [CrossRef]

21. Choi, W.; Sarkar, T.K. Minimum norm property for the sum of the adaptive weights for a direct data domain least squares algorithm. IEEE Trans. Antennas Propag. 2006, 54, 1045-1050. [CrossRef]

22. Cristallini, D.; Burger, W. A robust direct data domain approach for STAP. IEEE Trans. Signal Process. 2012, 60, 1283-1294. [CrossRef]

23. Weiss, M. Analysis of some modified cell averaging CFAR processors in multiple-target simulations. IEEE Trans. Aerosp. Electron. Syst. 1982, 18, 102-114. [CrossRef]

24. Xu, J.; Liao, G.; So, H.C. Space-Time Adaptive Processing with Vertical Frequency Diverse Array for Range-Ambiguous Clutter Suppression. IEEE Trans. Geosci. Remote Sens. 2016, 54, 5352-5364. [CrossRef]

25. Lan, L.; Liao, G.; Xu, J.; Zhang, Y.; Liao, B. Transceive Beamforming with Accurate Nulling in FDA-MIMO Radar for Imaging. IEEE Trans. Geosci. Remote Sens. 2020, 58, 4145-4159. [CrossRef]

26. Lan, L.; Xu, J.; Liao, G.; Zhang, Y.; Fioranelli, F.; So, H.C. Suppression of Mainbeam Deceptive Jammer with FDA-MIMO Radar. IEEE Trans. Veh. Technol. 2020, 69, 11584-11598. [CrossRef]

27. Xiang, T.S.; Wang, L.; Cao, Z.X.; Zhu, D.Y. Doppler-estimation based methods for airborne ISAR imaging of non-cooperative ship targets: Demonstration and analysis. In Proceedings of the IEEE international Radar symposium (IRS), Prague, Czech Republic, 28-30 June 2017; pp. 1-10. 